\title{
ᄀ PETEX
}

\section{ᄀ PETEX}

NEW IS POWER

\section{COMPOSITE}

REINFORCEMENT FABRIC

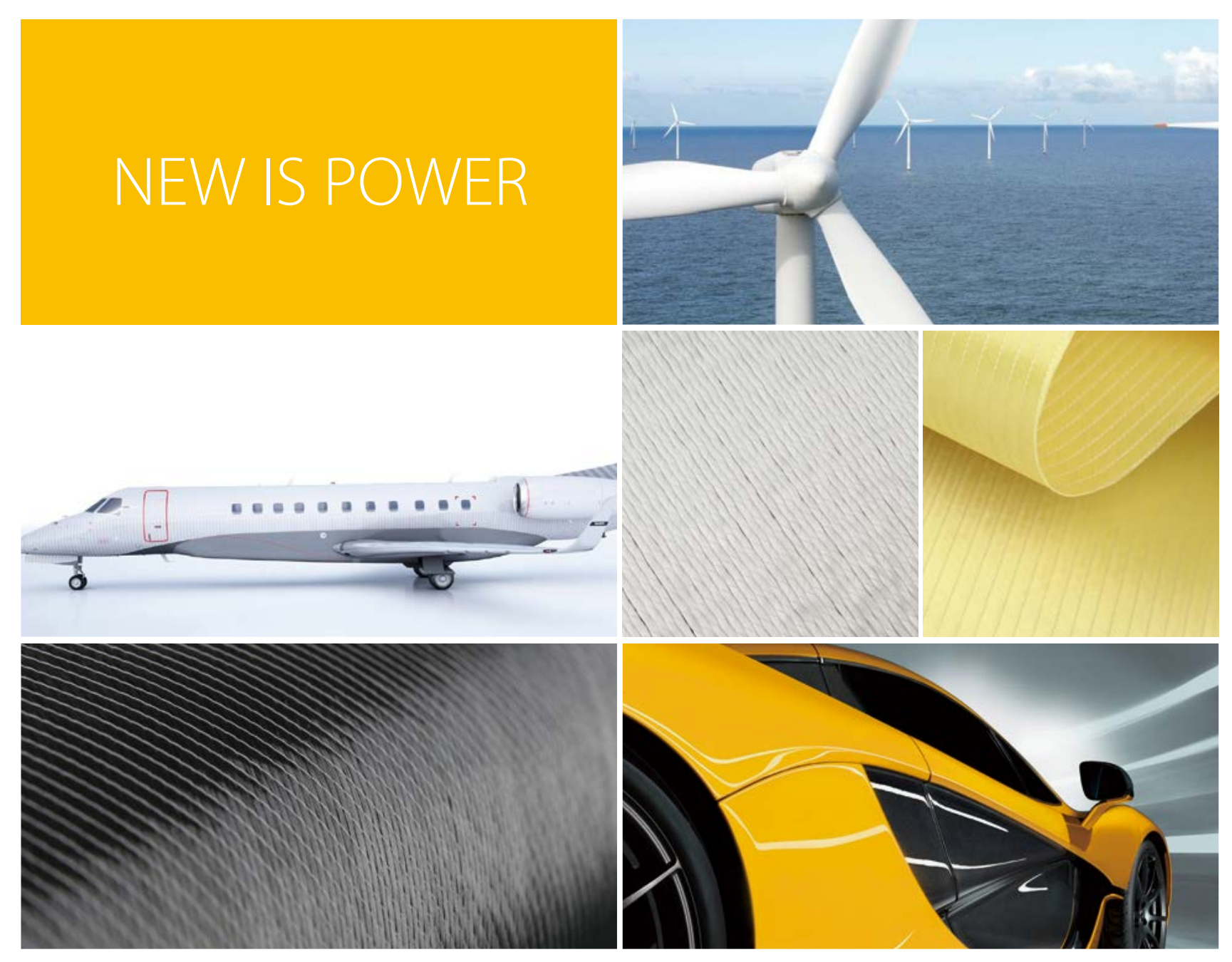




\section{PGTEX MILESTONES}

Developed the first glass fiber fabric
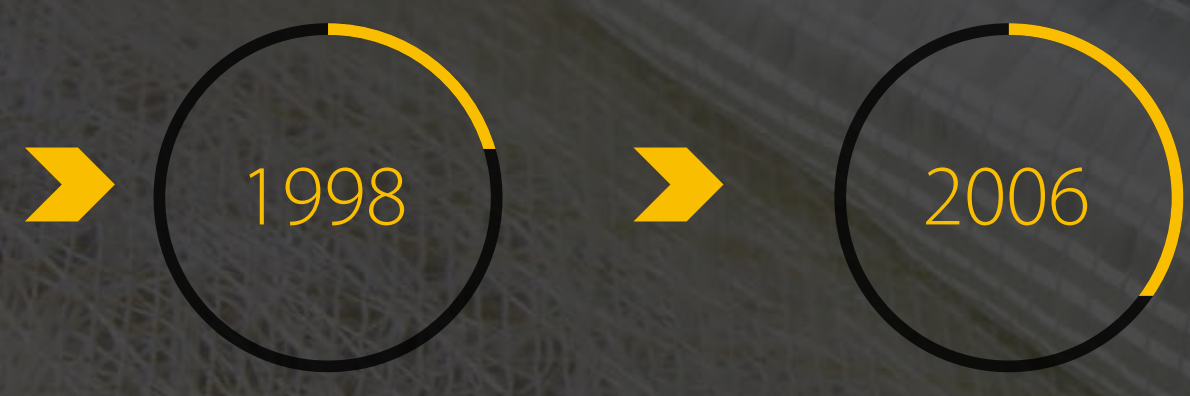

Developed the first carbon fiber fabric

est eq
2008 PGTEX reinforcement

fabric for wind power was

qualified by GLand $L R$ $1^{\text {st }}$ Generation PGTEX

carbon fiber multiaxial

machines with own design

STEP BY STEP

AS WE GROW

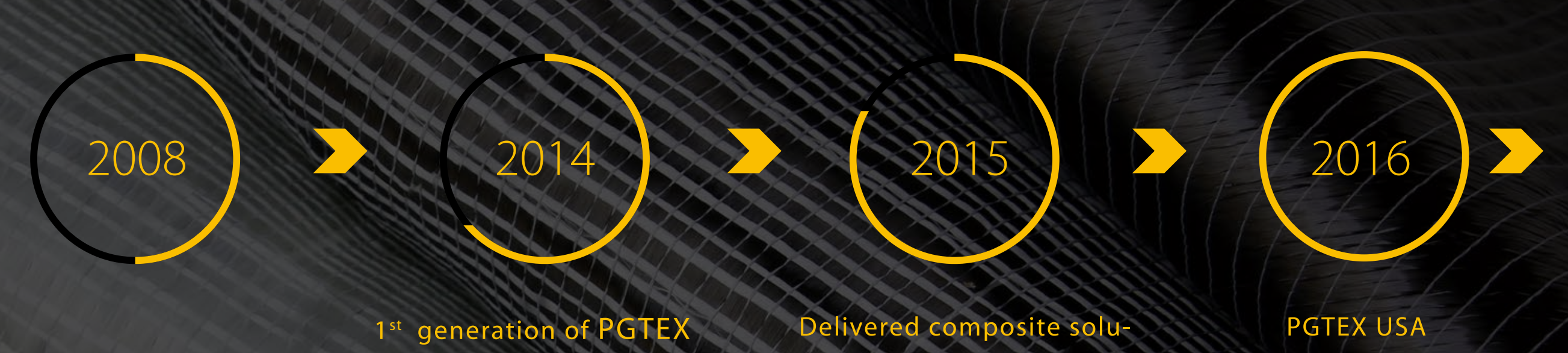

high speed multiaxial ma- tion for modular electricity

chines with own design.

bus

PGTEX USA 


\section{PGTEX \\ Global Footprint}

\section{Fabrics facilities:}

Asia-Changzhou/China,

America-EI Paso/USA,

EMEA-coming in 2018

\section{Roving facilities:}

Asia-Chongqing/China,

EMEA-Bahrain,

America-Brazil

El Paso, USA
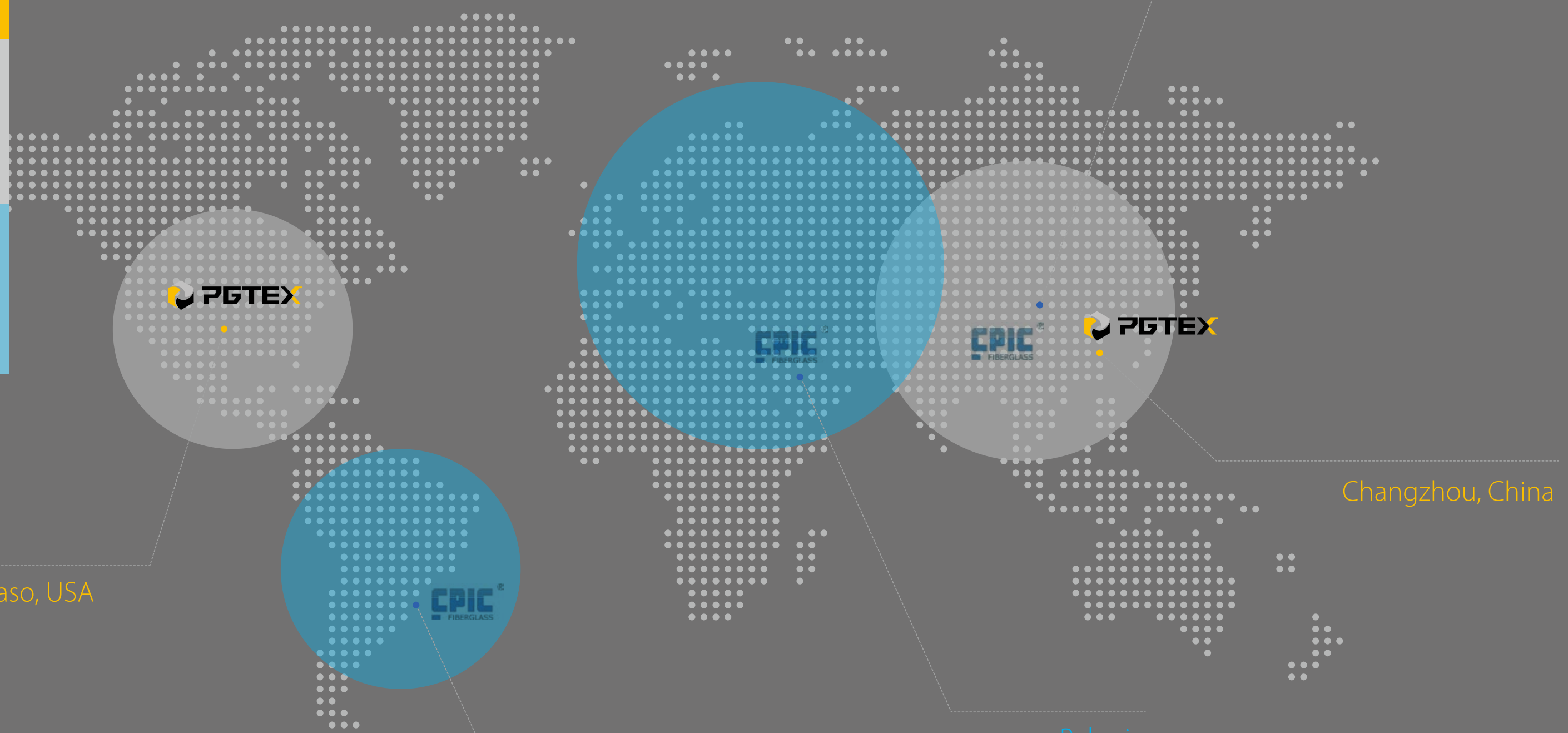

THINK GLOBALLY

MAKE GLOBALLY 


\section{PGTEX PARTNERS}

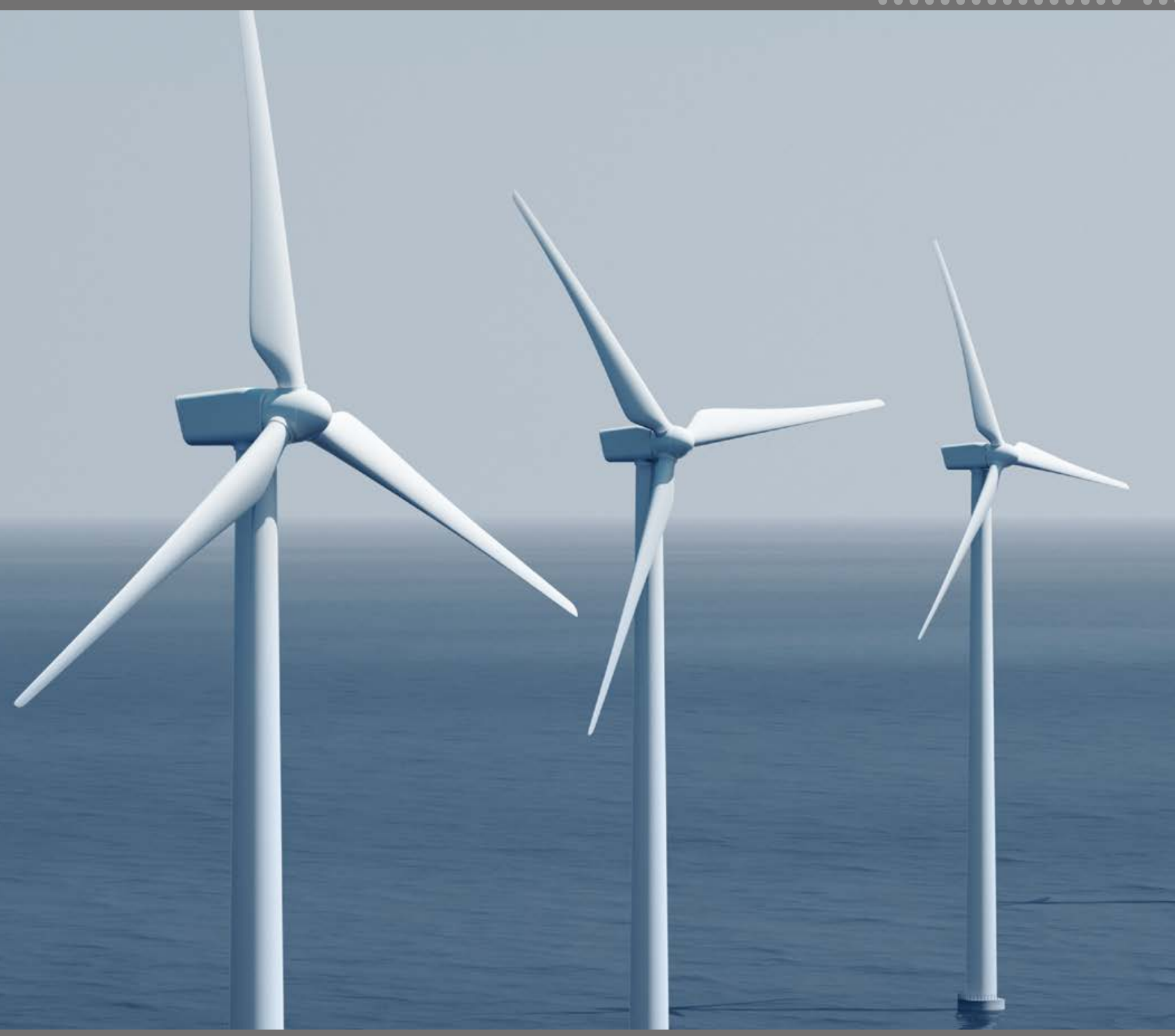

GMITIt SIEMENS

VARIOUS INDUSTRY

APPLICATIONS 


\section{PGTEX REINFORCEMENTS}

Aramid Fiber Fabric

Carbon Fiber Fabric

Glass Fiber Fabric

Hybrid Fiber Fabric
Naming Rule of High Performance Warp-Knitted Multiaxial Fabric
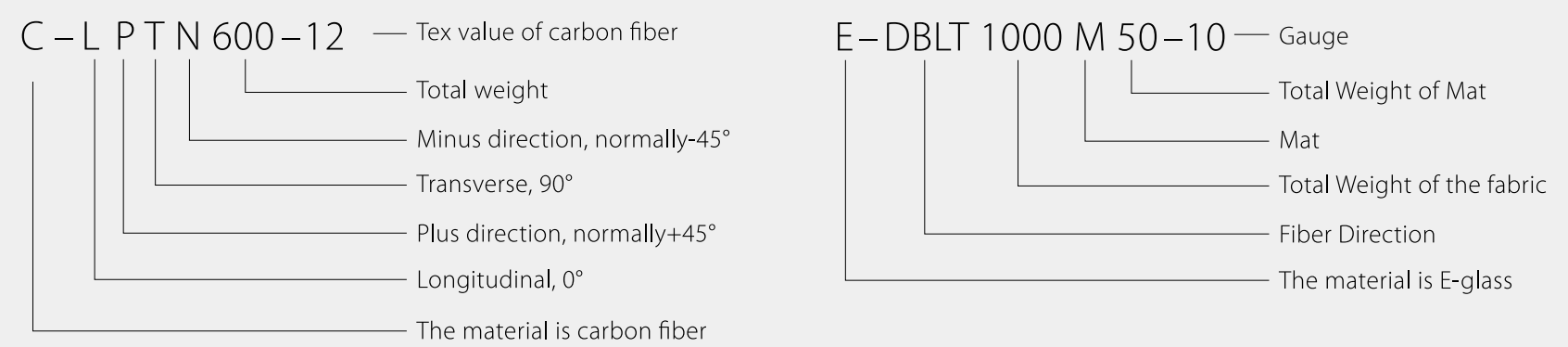

Naming Rule of High Performance Woven Fabric

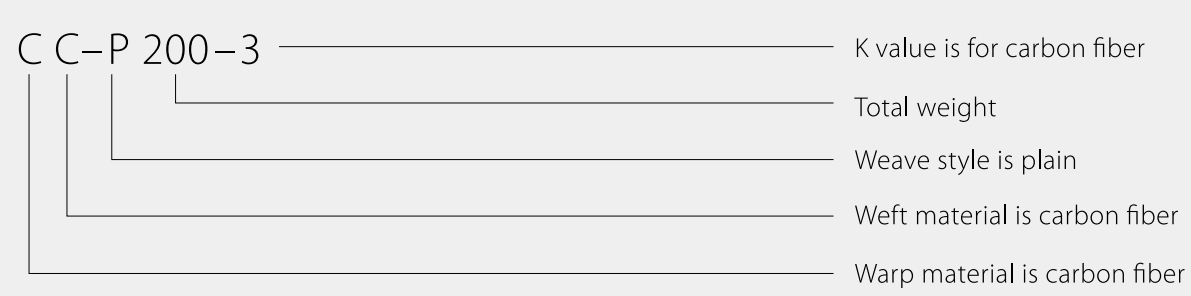

\section{Material discription:}

\begin{tabular}{|ll|ll|}
\hline E & E-glass & A & Aramide \\
\hline TM/TM+/H/HMG/E7 & H-glass & PE & UHMWPE \\
\hline S1/HT & S-glass & $V$ & Vectran \\
\hline C & Carbon & & \\
\hline
\end{tabular}

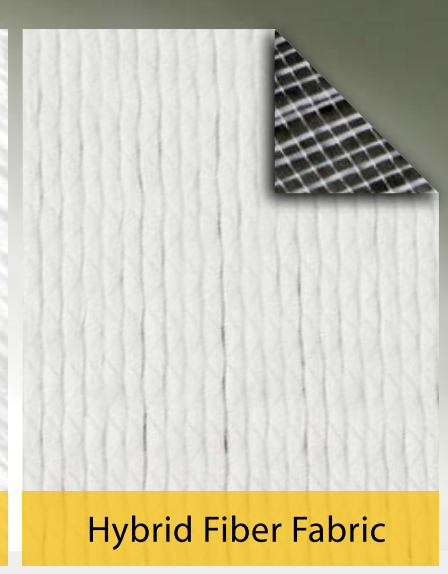

Structure diagram for high performance multiaxial warp-knitted fabric:

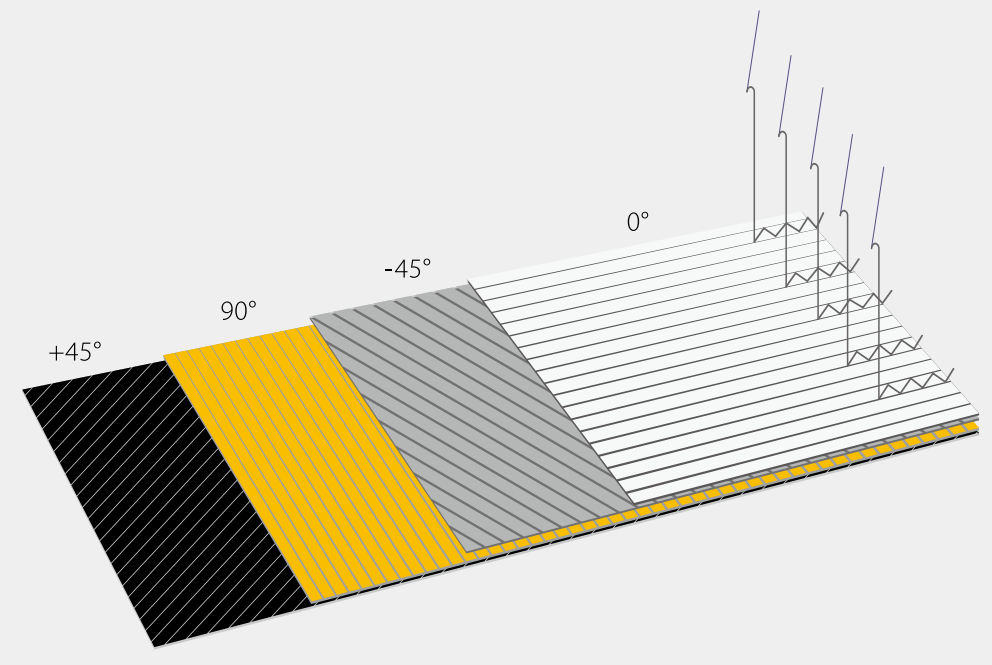

Fiber Direction

\begin{tabular}{ll} 
L & Longitudinal \\
T & Transverse \\
LT & $0^{\circ} / 90^{\circ}$ Biaxial \\
DB & $\pm 45^{\circ}$ Double Bias or $\left( \pm 30^{\circ} \sim \pm 895^{\circ}\right)$ \\
DBL & $0^{\circ} \pm 45^{\circ}$ Triaxial \\
DBT & $90^{\circ} \pm 45^{\circ}$ Triaxial \\
DBLT & $0^{\circ} / 45^{\circ} / 45^{\circ} / 90^{\circ}$ Quadriaxial \\
\hline
\end{tabular}

Structure diagram for high performance woven fabric:

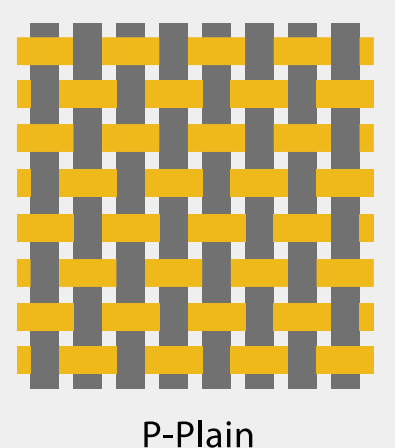

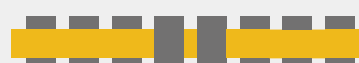

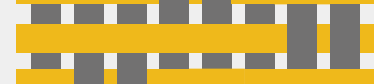

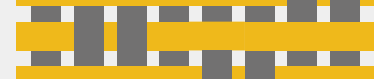

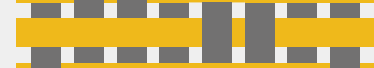

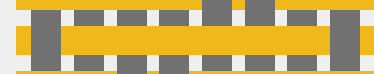

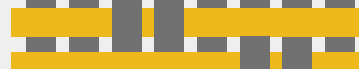

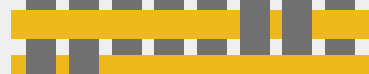

S-Satin

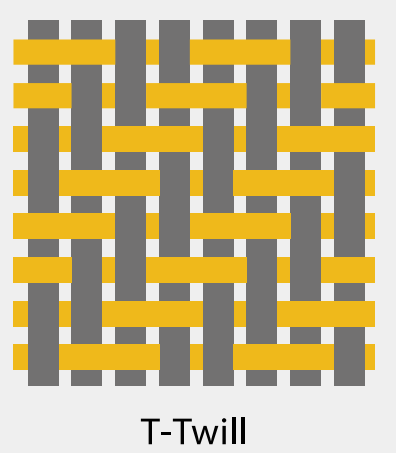




\section{PGTEX REINFORCEMENTS}

\section{Material}

Multiaxial Fabrics

Woven Fabrics

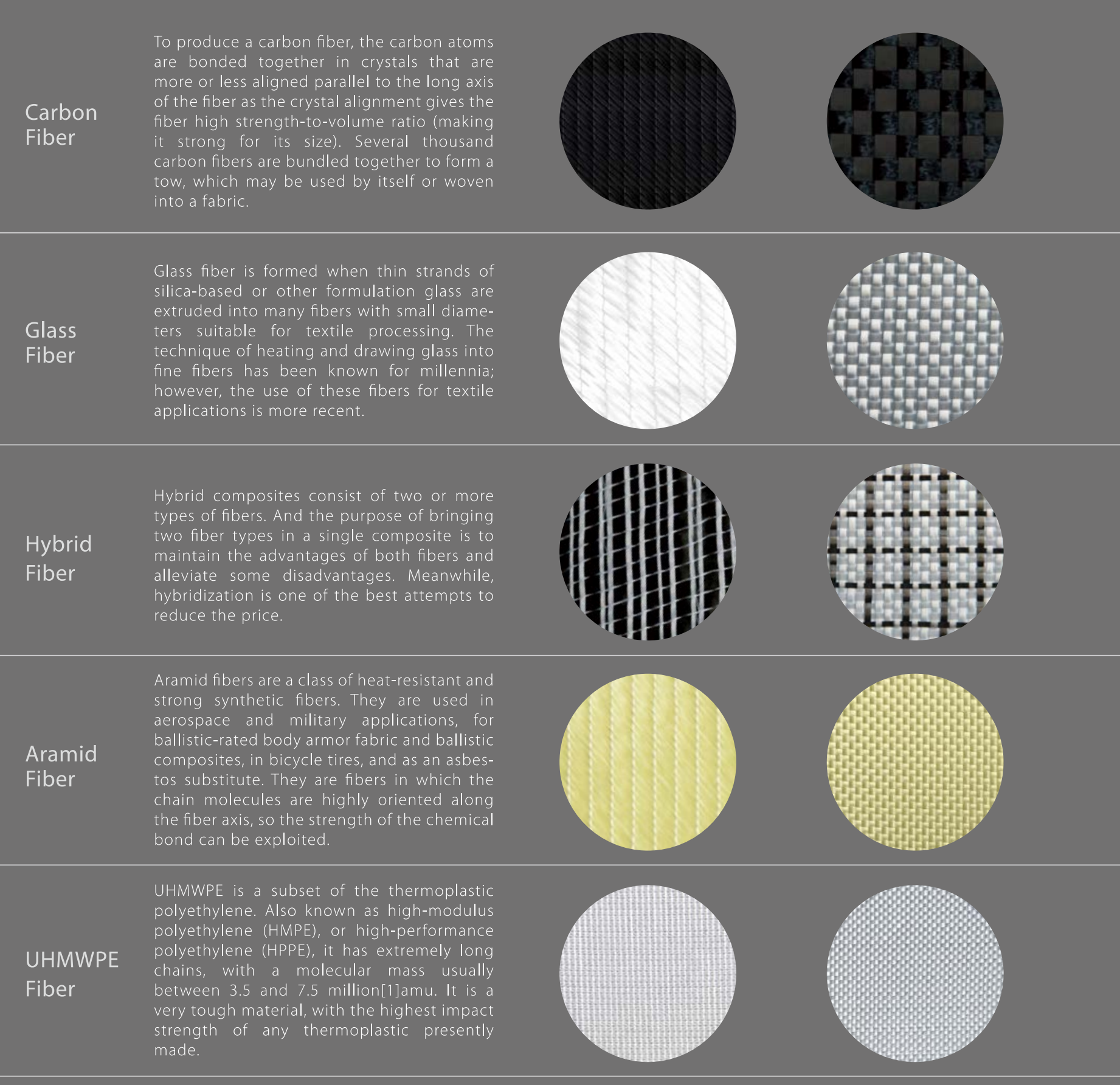

\section{VTE ATEL VALUE CHAIN}

\section{Integrating The Whole Value Chain}

Composite markets based on fiber-reinforced materials pose their own challenges. PGTEX address these challenges by partnership with the customers in different markets and seeing their pain-points. We integrated the key players along the value chain to provide the total composite solutions to our customers, from roving, designing, testing to manufacturing.

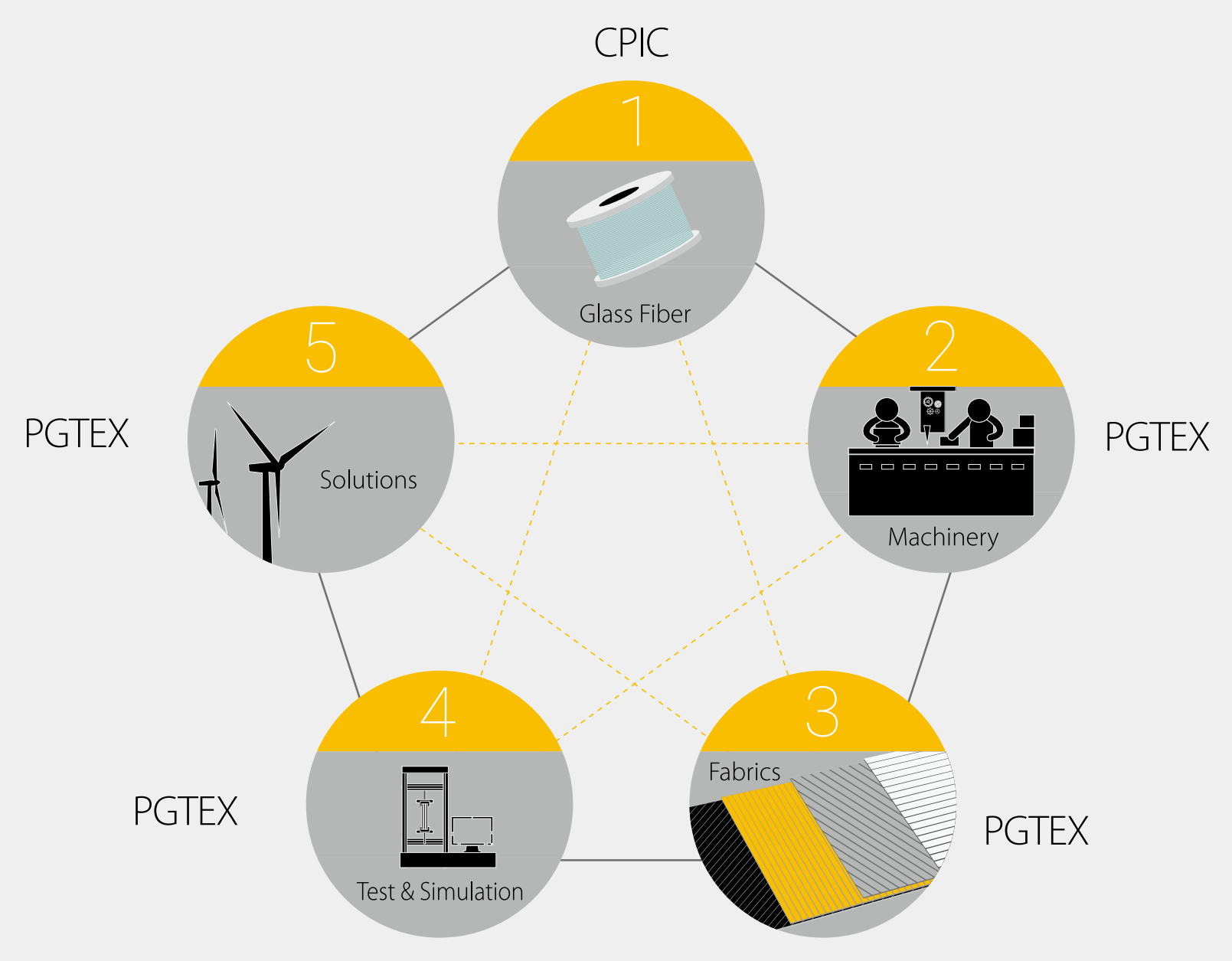




\section{PGTEX}

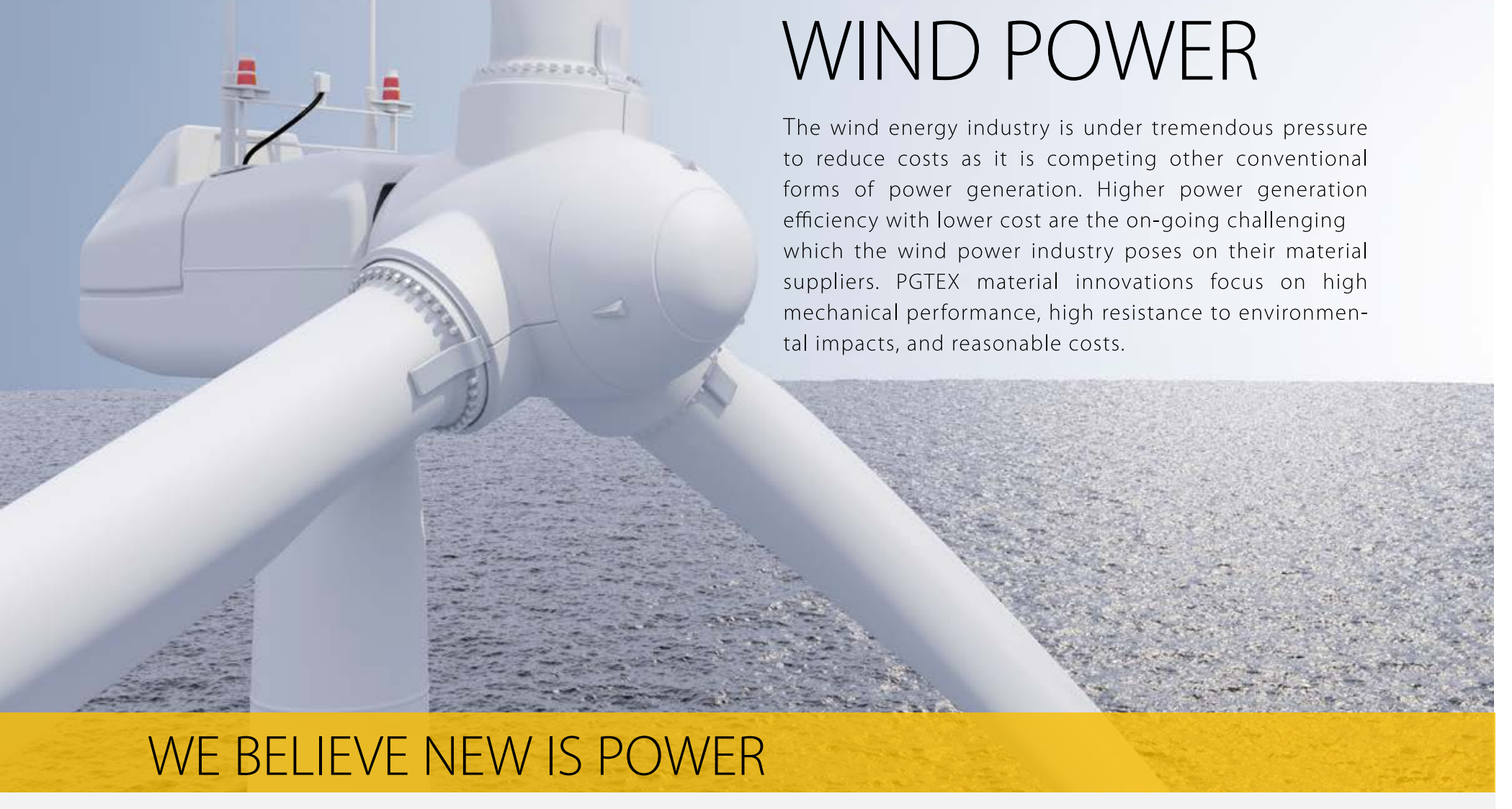

WE BELIEVE NEW IS POWER

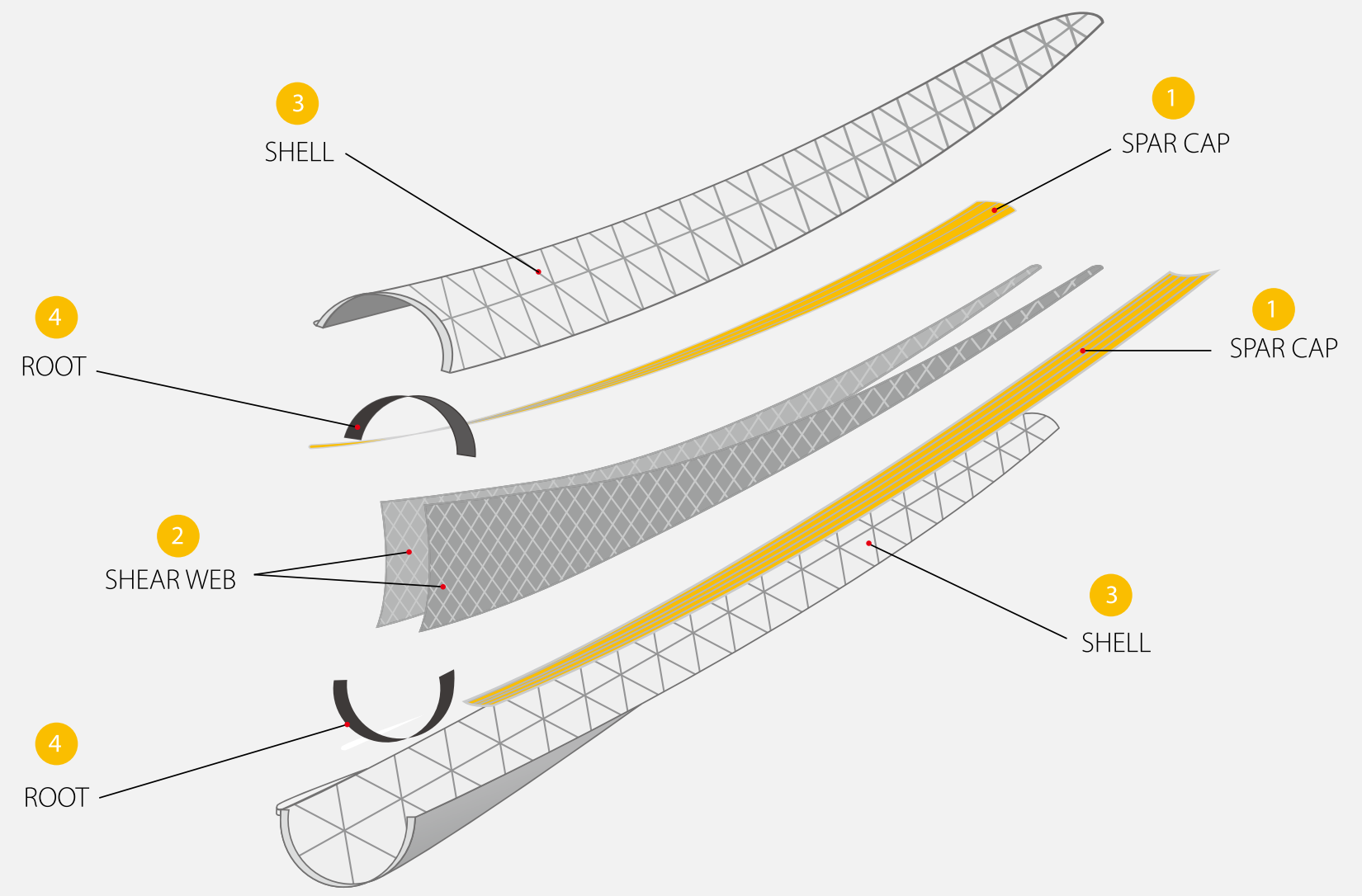

\section{(1) SPAR CAP}

\begin{tabular}{|c|c|c|c|c|c|c|c|}
\hline $\begin{array}{l}\text { Fabric } \\
\text { Specification }\end{array}$ & $\begin{array}{c}\text { Total Weight } \\
\left(\mathrm{g} / \mathrm{m}^{\prime}\right)\end{array}$ & $0^{\circ}$ & $90^{\circ}$ & $+45^{\circ}$ & $-45^{\circ}$ & $\begin{array}{l}\text { Mat } \\
\left(\mathrm{g} / \mathrm{m}^{3}\right)\end{array}$ & $\begin{array}{c}\text { Stiching Yarn } \\
\left(\mathrm{g} / \mathrm{m}^{\prime}\right)\end{array}$ \\
\hline E-L1200 & 1208 & 1150 & 50 & ' & 1 & 1 & $\leq 9$ \\
\hline TMLL1200 & 1208 & 1150 & 50 & ' & I & I & $\leq 9$ \\
\hline TM-L1250 & 1248 & 1196 & 40 & ' & , & ' & $\leq 12$ \\
\hline E-L1200M50 & 1258 & 1150 & 50 & 1 & 1 & 50 & $\leq 9$ \\
\hline TM-L1200M50 & 1258 & 1150 & 50 & I & / & 50 & $\leq 9$ \\
\hline
\end{tabular}

(2) SHEARWEB

\begin{tabular}{cccccccc}
$\begin{array}{c}\text { Fabric } \\
\text { Specification }\end{array}$ & $\begin{array}{c}\text { Total Weight } \\
\left(g / \mathrm{m}^{\prime}\right)\end{array}$ & $0^{\circ}$ & $90^{\circ}$ & $+45^{\circ}$ & $-45^{\circ}$ & $\begin{array}{c}\text { Mat } \\
\left(\mathrm{g} / \mathrm{m}^{\prime}\right)\end{array}$ & $\begin{array}{c}\text { Stiching Yarn } \\
\left(\mathrm{g} / \mathrm{m}^{\circ}\right)\end{array}$ \\
\hline E-DB800 & 813 & 2 & 2 & 401 & 401 &, & $\leq 8$ \\
E-DB1000 & 973 & 1 & 30 & 468 & 468 &, & $\leq 8$
\end{tabular}

\section{(3) SHELL}

\begin{tabular}{|c|c|c|c|c|c|c|c|}
\hline $\begin{array}{l}\text { Fabric } \\
\text { Specification }\end{array}$ & $\begin{array}{l}\text { Total Weight } \\
\left(\mathrm{g} / \mathrm{m}^{\prime}\right)\end{array}$ & $0^{\circ}$ & $90^{\circ}$ & $+45^{\circ}$ & $-45^{\circ}$ & $\begin{array}{l}\text { Mat } \\
\left(g / m^{\prime}\right)\end{array}$ & $\begin{array}{c}\text { Stiching Yarn } \\
\left(g / m^{\prime}\right)\end{array}$ \\
\hline E-DB800 & 813 & 2 & 2 & 401 & 401 & / & $\leq 8$ \\
\hline E-DBL750 & 752 & 142 & I & 301 & 301 & 1 & $\leq 12$ \\
\hline E-DBL750M30 & 782 & 142 & 1 & 300 & 300 & 30 & $\leq 12$ \\
\hline E-DB.800 & 808 & 236 & I & 280 & 280 & I & $\leq 12$ \\
\hline E-DBL800M30 & 838 & 236 & I & 280 & 280 & 30 & $\leq 12$ \\
\hline E-DB.1870 & 876 & 567 & 1 & 200 & 200 & I & $\leq 13$ \\
\hline E-DBL.870M30 & 906 & 567 & 1 & 200 & 200 & 30 & $\leq 13$ \\
\hline E-DBLL1200 & 1177 & 567 & I & 301 & 301 & I & $\leq 10$ \\
\hline E-DBL1200M30 & 1208 & 567 & / & 301 & 301 & 30 & $\leq 10$ \\
\hline E-DBLL1215 & 1218 & 709 & I & 250 & 250 & I & $\leq 10$ \\
\hline E-DBLL1215M30 & 1248 & 709 & 1 & 250 & 250 & / & $\leq 10$ \\
\hline E-DBLL1250 & 1250 & 640 & $\leq 10$ & 301 & 301 & I & $\leq 8$ \\
\hline E-L1200 & 1208 & 1150 & 50 & , & 1 & I & $\leq 9$ \\
\hline E-L1200M50 & 1258 & 1150 & 50 & I & 1 & 50 & $\leq 9$ \\
\hline TM-LL1200 & 1208 & 1150 & 50 & , & 1 & I & $\leq 9$ \\
\hline TM-L1250 & 1248 & 1196 & 40 & 1 & 1 & I & $\leq 12$ \\
\hline
\end{tabular}

\section{(4) ROOT}

\begin{tabular}{|c|c|c|c|c|c|c|c|}
\hline $\begin{array}{l}\text { abric } \\
\text { Specification }\end{array}$ & $\begin{array}{l}\text { Total Weight } \\
\left(g / \mathrm{m}^{\prime}\right)\end{array}$ & $0^{\circ}$ & $90^{\circ}$ & $+45^{\circ}$ & $-45^{\circ}$ & $\begin{array}{l}\text { Mat } \\
\left(\mathrm{g} / \mathrm{m}^{3}\right)\end{array}$ & $\begin{array}{c}\text { Stiching Yarn } \\
\left(g / \mathrm{m}^{\prime}\right)\end{array}$ \\
\hline 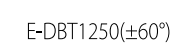 & 1250 & $\leq 10$ & 640 & 301 & 301 & 1 & $\leq 8$ \\
\hline E-DBT1 2200 & 1177 & ' & 567 & 301 & 301 & 1 & $\leq 9$ \\
\hline E-DBT1215 & 1218 & 1 & 709 & 250 & 250 & 1 & $\leq 10$ \\
\hline
\end{tabular}




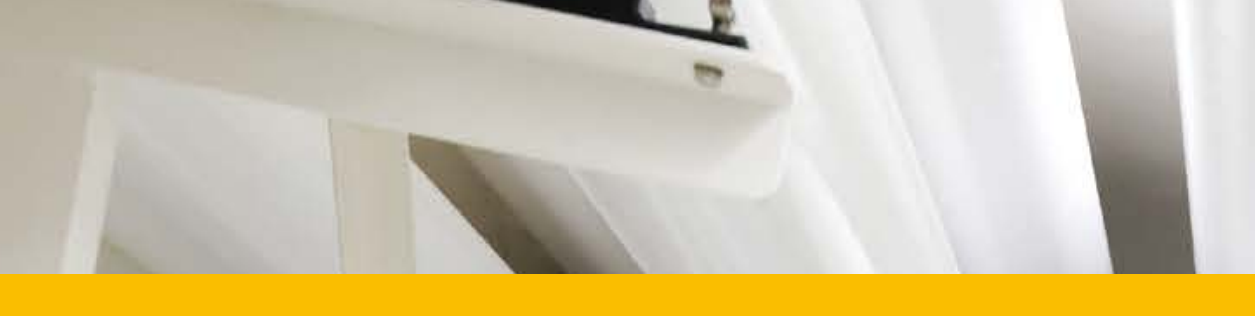

PGTEX

INDUSTRIES

STREAMLINEYOUR EFFICIENCY
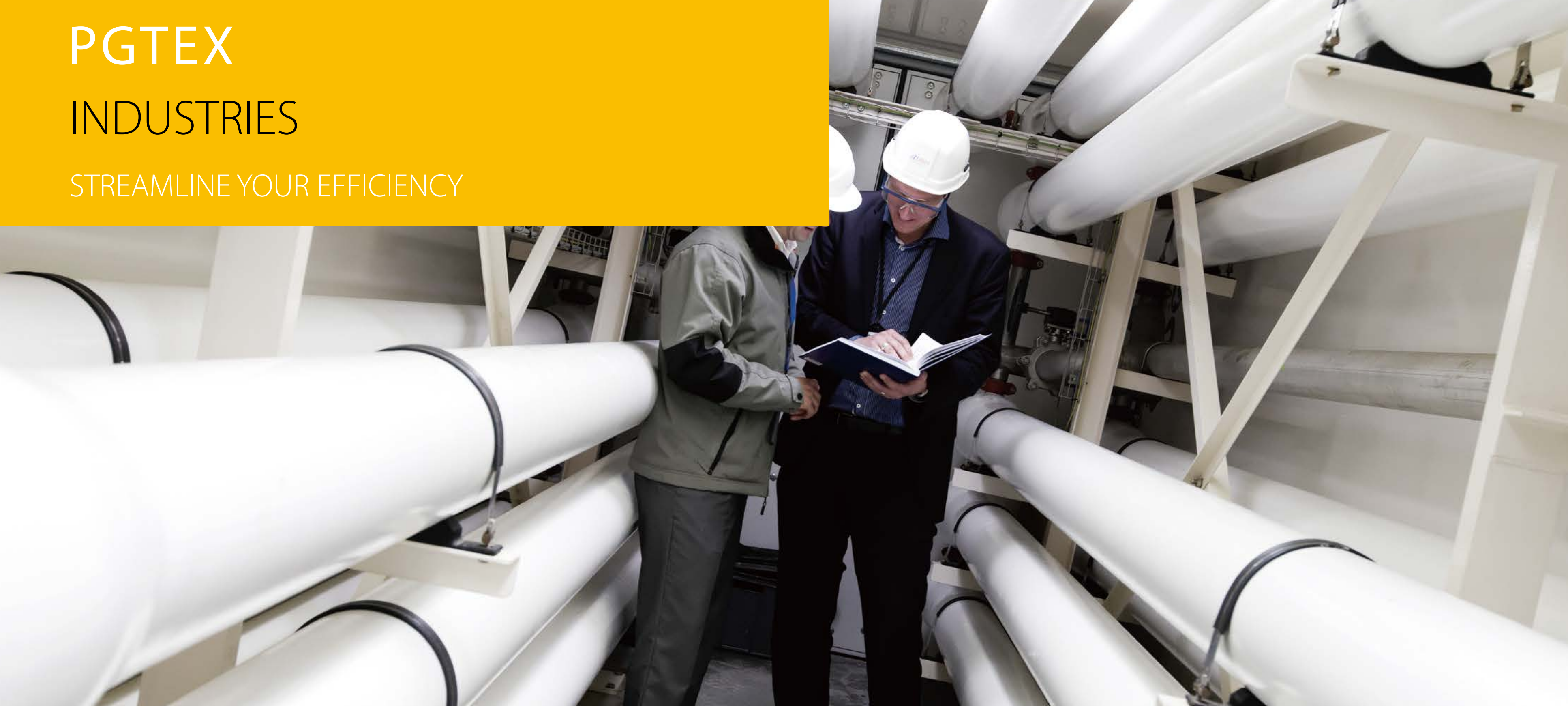

PIPELINE

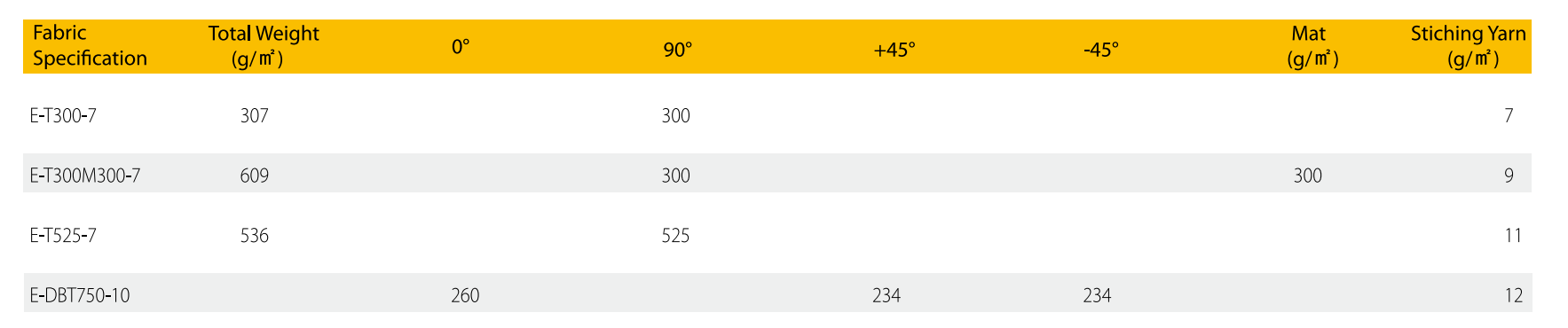

PULTRUSION

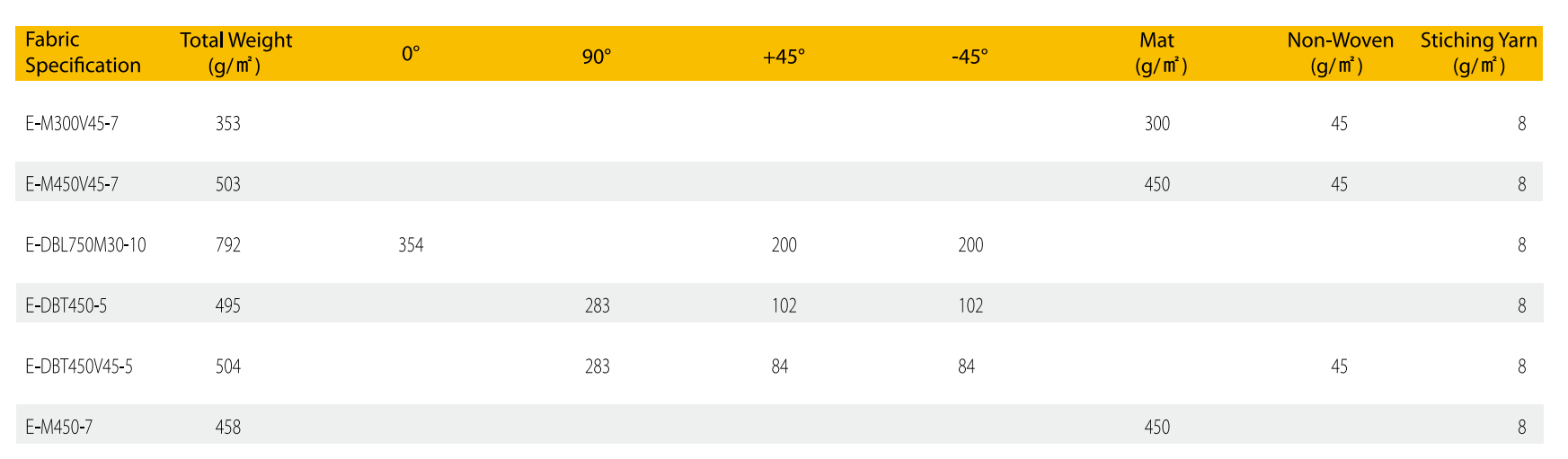




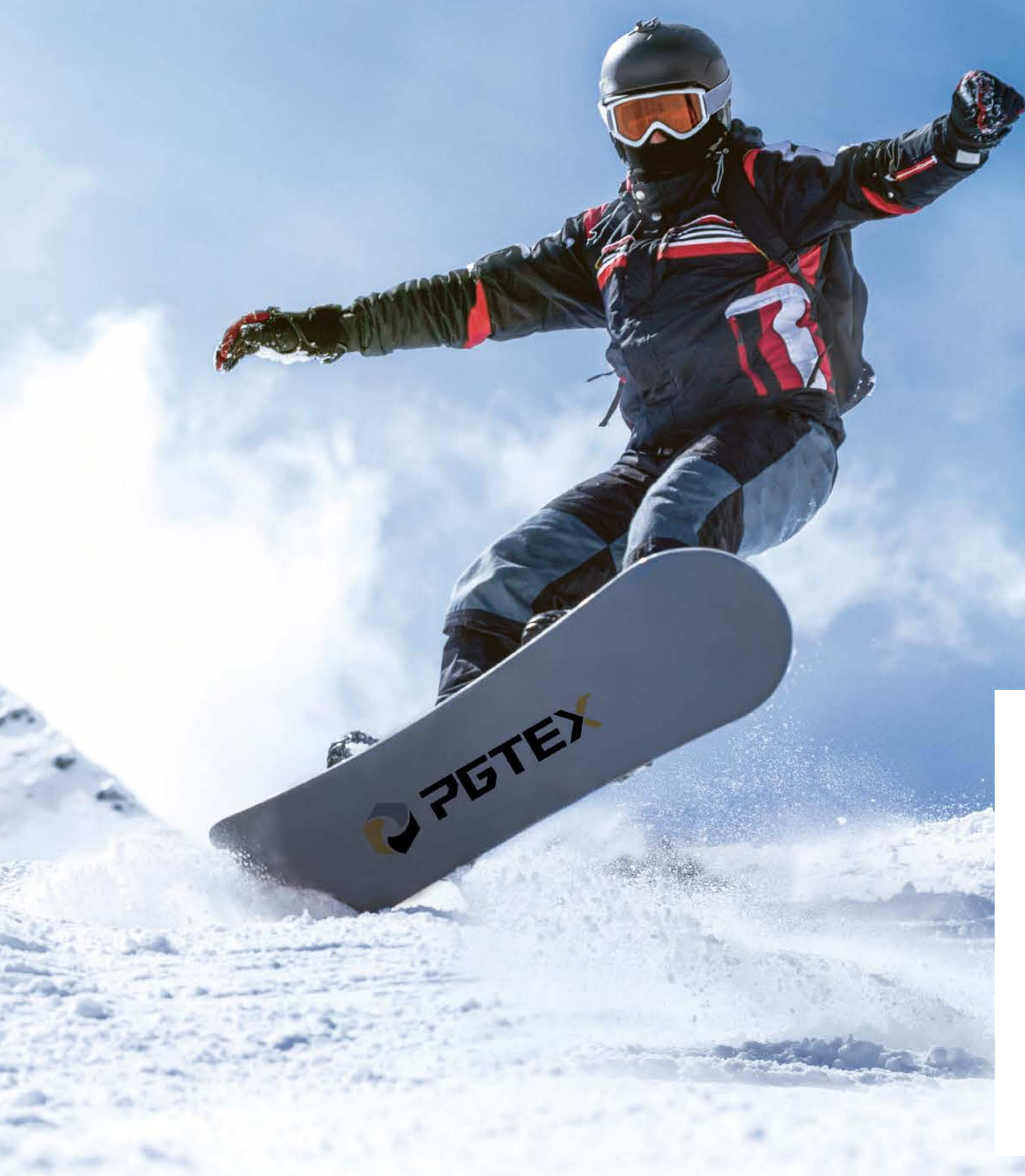

\section{PGTEX \\ SPORTS}

MAKE YOUR ACTION

MORE EXCITING

Reaching limits of "Higher, Faster and Stronger" is an eternal mission in

sports and leisure activities. The sports players are always asking for

lighter and stronger sport equipment to deliver better racing results.

So sports are very specialized markets. They pose challenges materia

suppliers to develop and manufacture materials with specific good

performance at reasonable costs.

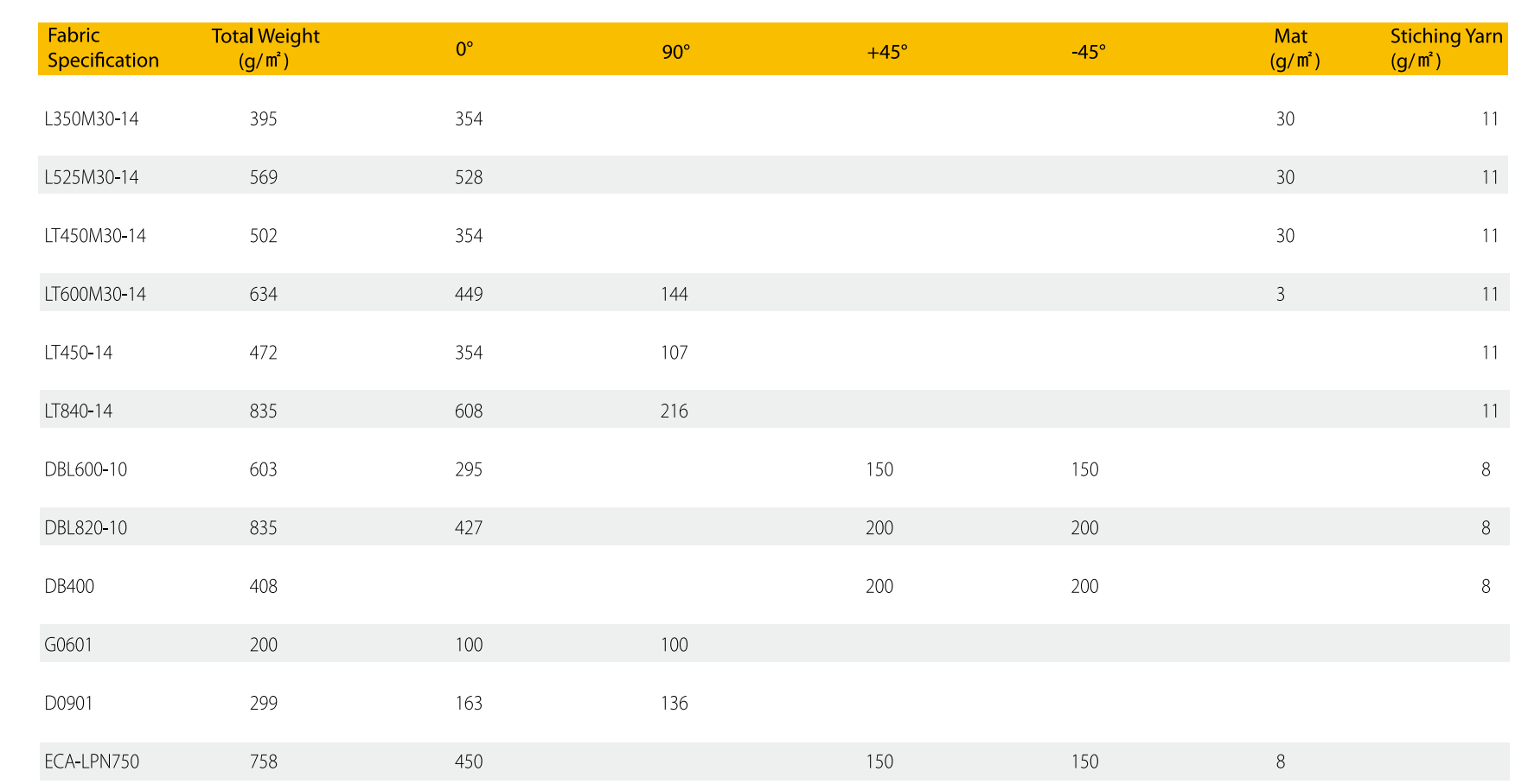




\section{PGTEX AWARD}

\section{PGTEX CULTURE}

- 2015 JEC Innovation Awards

- National Hi-new Tech company

- National Torch Program

- State Innovation Award

- National Manufacture Center of Carbon Fiber Knitted Reinforcement

- National Famous Trade Mark

- Drafted 3 National Standards for Composite

- State R\&D Center of Advanced Fiber Reinforcements

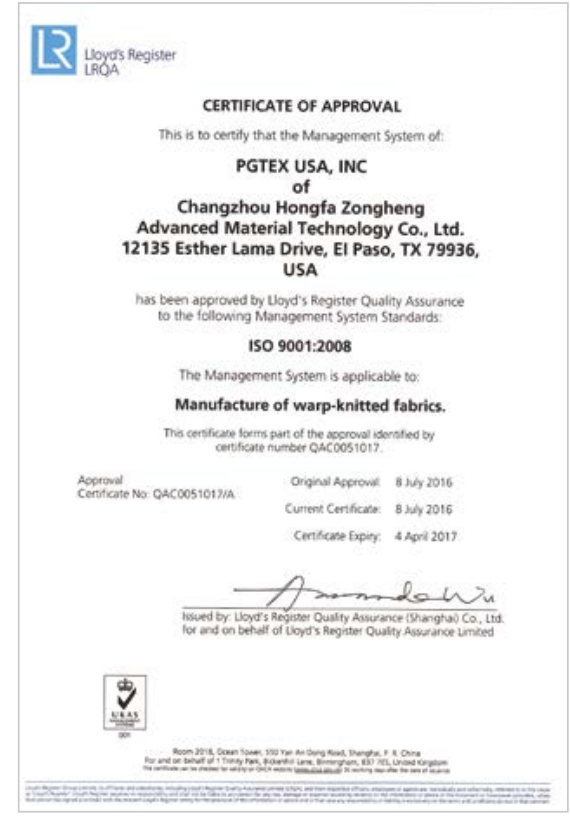

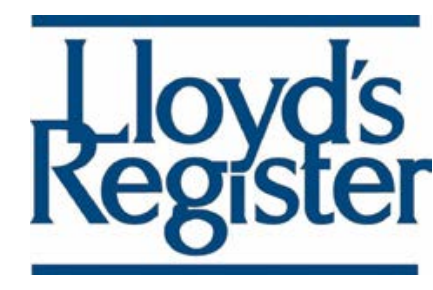

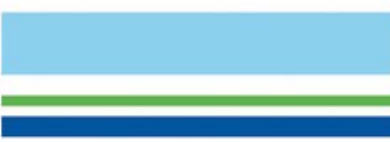

DNV·GL
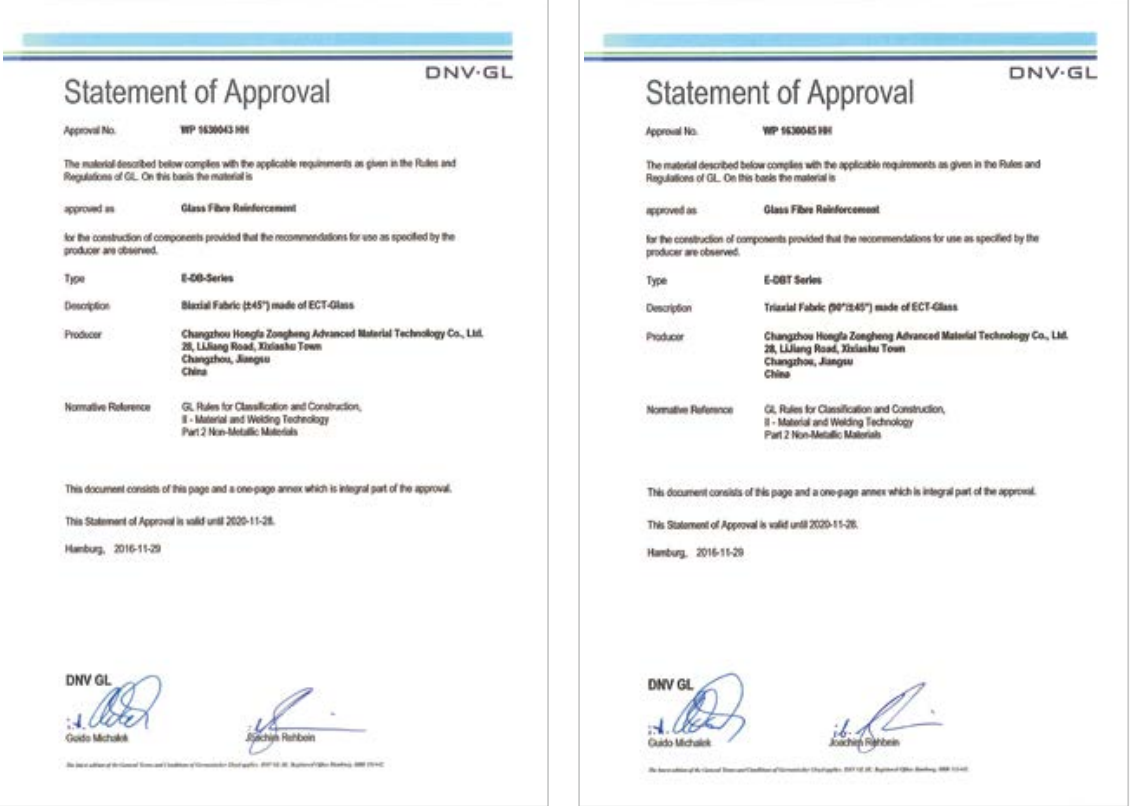

\section{Vision:}

Build to Last, Manage to Sustain

\section{Mission:}

Delivering light-weight \& low-cost composites solutions to customers 